\title{
Coexistence of a novel CCNY-ALK and ATIC-ALK double-fusion in one patient with ALK-positive NSCLC and response to crizotinib: a case report
}

\author{
Xuan Wu ${ }^{1 \#}$, Hanqiong Zhou ${ }^{1 \#}$, Zhen $\mathrm{He}^{1 \#}$, Zhe Zhang ${ }^{1}$, Wen Feng ${ }^{2}$, Jiuzhou Zhao ${ }^{3}$, Haiyang Chen ${ }^{1}$, \\ Shuai Wang', Wei Wang ${ }^{4}$, Qiming Wang ${ }^{1}$ \\ ${ }^{1}$ Department of Internal Medicine, Henan Cancer Hospital Affiliated to Zhengzhou University, Zhengzhou, China; ${ }^{2}$ Department of Pathology, \\ Henan Cancer Hospital Affiliated to Zhengzhou University, Zhengzhou, China; ${ }^{3}$ Department of Molecular Pathology, Henan Cancer Hospital \\ Affiliated to Zhengzhou University, Zhengzhou, China; ${ }^{4}$ Henan Medical Association, Zhengzhou, China \\ \#These authors contributed equally to this work. \\ Correspondence to: Qiming Wang, MD, PhD. Department of Internal Medicine, Henan Cancer Hospital Affiliated to Zhengzhou University, 127 \\ Dong Ming Road, Zhengzhou 450008, China. Email: qimingwang1006@126.com; Wei Wang, MD. Henan Medical Association, 45 Wei Wu Road, \\ Zhengzhou, Henan 450008, China. Email: weiwang68@126.com.
}

\begin{abstract}
Anaplastic lymphoma kinase (ALK) rearrangement, one of the common oncogene rearrangements in the mutational history of lung adenocarcinoma, occurs in approximately $5 \%$ of non-small cell lung cancer (NSCLC) patients who could be effectively treated with ALK tyrosine kinase inhibitors (TKIs). The earlier phase III PROFILE 1014 study has shown that crizotinib, a first-generation ALK-TKI, significantly improved progression-free survival (PFS) compared with platinum-based chemotherapy in patients with previously untreated advanced ALK-positive NSCLC. Thus, clinicians must screen potential candidates for this driver alteration to guide ALK inhibitor therapy with a molecular testing platform capable of capturing all ALK fusions. Echinoderm microtubule-associated proteins, including the EML4 gene, are the most common ALK rearrangement partner. With the widespread use of the next-generation sequencing (NGS) techniques, which could approach enable the simultaneous screening of multiple genetic alterations, increasingly ALK rearrangement partners have been documented. However, the concurrent two ALK rearrangements within the same patient have rarely previously been reported. Here, we describe a novel CCNY-ALK (C1:A20) and ATIC-ALK (A7:A20), coexisting in the same case with poorly differentiated NSCLC and providing evidence of its sensitivity to ALK inhibitors. The newly identified rearrangement partners can be added to the list of ALK rearrangements that occurred in ALK-positive NSCLC, as it could lead to prolonged disease control. Also, while different ALK rearrangement variants might bring differing clinical outcomes, we discuss the impact of the co-mutations of these two ALK rearrangements on the sensitivity to ALK inhibitors. However, the impact of co-mutations on the pathogenesis of NSCLC should be further studied to supply more theoretical insight that co-mutations present for personalized anti-cancer therapy.
\end{abstract}

Keywords: Anaplastic lymphoma kinase (ALK); non-small cell lung cancer (NSCLC); cyclin Y (CCNY); 5-aminoimidazole-4-carboxamide ribonucleotide formyltransferase/IMP cyclohydrolase (ATIC); case report

Submitted Aug 26, 2020. Accepted for publication Oct 28, 2020.

doi: $10.21037 /$ tlcr-20-1049

View this article at: http://dx.doi.org/10.21037/tlcr-20-1049 


\section{Introduction}

Anaplastic lymphoma kinase (ALK)-positive non-small cell lung cancer (NSCLC), which results in dysregulation and incorrect signaling through the ALK kinase domain, comprises approximately $4 \%$ of all lung cancer and carries an excellent prognosis with ALK tyrosine kinase inhibitors (TKIs) $(1,2)$. ALK rearrangement is usually taken into consideration when deciding on the administration of ALK-TKIs for the management of NSCLC. With the popularity of next-generation sequencing (NGS) technology, besides EML4-ALK, other rearrangement types sensitive to ALK-TKIs, including SPECC1L-ALK, PLEKHM2-ALK, and WDPCP-ALK, have been detected (3-5); however, the case with ALK double rearrangement is rare (6). Also, considering the disparate clinical outcome of different ALK variants, it is imperative to test the response of both double and new types of rearrangement to ALK-TKIs. Some previous reports have confirmed that patients with double ALK rearrangements may be sensitive to crizotinib. Here, we present the case of an ALKpositive poorly differentiated NSCLC patient with cyclin $\mathrm{Y}$ (CCNY)-ALK and 5-aminoimidazole-4-carboxamide ribonucleotide formyltransferase/IMP cyclohydrolase (ATIC)-ALK, treated with crizotinib and achieved a partial response for at least six months. This patient with the novel double-ALK rearrangement responded to crizotinib and a novel CCNY-ALK rearrangement was detected. In the CCNY-ALK rearrangement, the breakpoint occurs in intron 1 of CCNY and in intron 19 of ALK, the promotor region of $\mathrm{CCNY}$ and the entire intracellular kinase domain of ALK was retained. This is the first paper to report the novel double-ALK rearrangement of CCNYALK and ATIC-ALK that respond to crizotinib treatment. We present the following article in accordance with the CARE reporting checklist (available at http://dx.doi. org/10.21037/tlcr-20-1049).

\section{Case presentation}

A 32-year-old man with a ten pack per year smoking history was admitted to our hospital for thoracic examination complaining of coughing and hemoptysis for more than one month. Chest computed tomography (CT) reveals a left hilar mass, measured $84 \mathrm{~mm} \times$ $53 \mathrm{~mm}$, accompanied by pulmonary atelectasis in association with pleural effusion, obstructive pneumonia, enlargement of the hilum of the left lung, and mediastinal lymph nodes (Figure 1). No metastases were found by magnetic resonance imaging (MRI) of the brain. CTguided pulmonary puncture pathology analysis showed poorly differentiated NSCLC (cT4N3M1a, stage Iva). Immunohistochemistry analysis was positive for thyroid transcription factor 1, CK, LCA, Vim, and $80 \%$ by Ki67, but negative for CK5/6, CK7, NapsinA, and P40 (Figure 2). Fluorescent in situ hybridization (FISH) was performed on the biopsy sample to identify the ALK rearrangement status, and ALK-positive NSCLC was confirmed with positive and atypical signals to seek more potential treatments in patients with NSCLC. Also, immunohistochemistry (ALK D5F3 antibody) reveals strong expression of ALK protein at the primary lung lesion (Figure 2). To further identify the rearrangement type of ALK gene, we evaluate the genetic alteration of a collected pathological tissue sample using the DNA-based NGS (Burning Rock, Guangzhou, China) with a gene panel comprising eight lung cancer-related genes (EGFR, ALK, ERBB2, BRAF, MET, ROS1, RET, and KRAS). The concurrent two ALK rearrangements are found, including a novel CCNY-ALK (C1:A20) and ATIC-ALK (A7:A20). Besides, no added driver events with robust transforming potential have been detected. For novel CCNY-ALK rearrangement, the first intron of CCNY and the 19th intron of ALK were broken and rearranged; an abundance rate of $16.64 \%$ was observed. The fusion sequence is GT GGGCAGTTGGAAACAGTAATCCAATCTACAGCA GATGGAGATTCAGTT<>AAGTGACGTCTAGGG GTGGGGGCGAGCTTTCACCATCGTGATGGAC ACTG. In the novel ATIC-ALK rearrangement, the 7th intron of ATIC and the 19th intron of ALK were broken and rearranged, with an abundance of $16.33 \%$. The fusion sequence is AGAGTGGGCTAGTGCATTACATAGGGT GGGAGCCAAACAGGAGCTGCGCC<>AACGCGGG AGGTGGAGTTTGCAGCGAGCCAAGATCGCACCA CTGCACTCC (Figure 3). Then the patient was given oral crizotinib $(250 \mathrm{mg}$, twice daily) as a first-line treatment. Clinical symptoms of dyspnea and cough were improved, and the size of lung mass decreased sharply a month later (Figure 1). Partial response was further confirmed after six months (Figure 1). There were no severe toxic effects during the whole crizotinib therapy. However, since then, the patient never came to the hospital again and lost to follow up in the hospital.

The Medical Ethics Committee approved this study of Henan Cancer Hospital of Zhengzhou University, and the participating patients gave consent. All procedures 

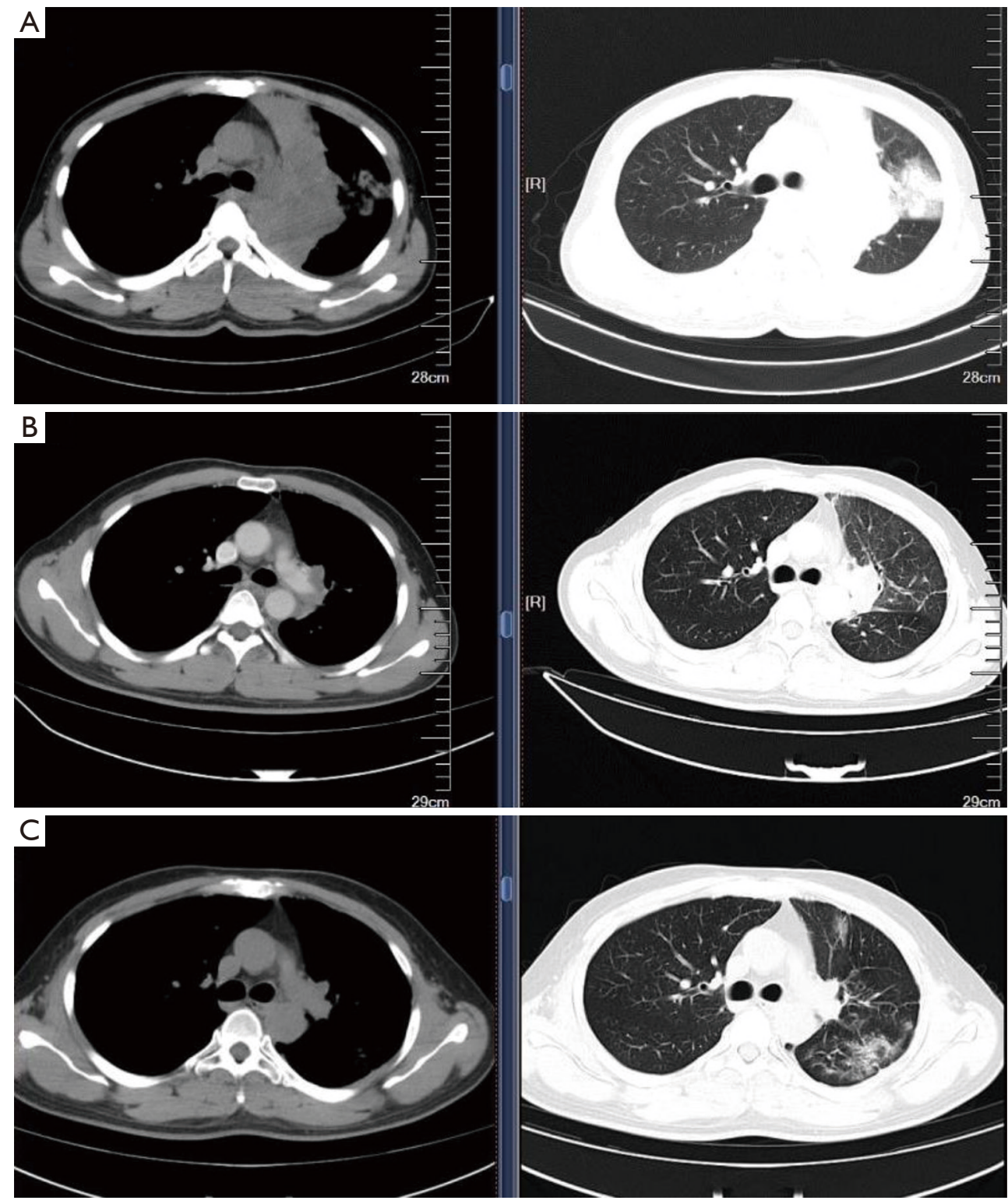

Figure 1 CT scans before and after therapy. (A) CT scans before crizotinib treatment. (B) CT scans after 1 month of treatment with crizotinib. (C) CT scans after 6 months of treatment with crizotinib (C). CT scans showed significant shrinkage of the lung mass together with mediastinal and hilar lymph nodes. CT, computed tomography.

performed in studies involving human participants were in accordance with the Helsinki Declaration (as revised in 2013). The patient gave written consent for his personal or clinical details, along with any identifying images to be published in this study. A copy of the written consent is available for review by the Editor of this journal. The case has not been reported before and is not being considered for publication elsewhere.

\section{Discussion}

The ALK gene is fused with several genes, which are essential in the constitutive activation of the chimeric protein and its subcellular localization. The $\mathrm{N}$-terminal rearrangement partner of the ALK gene provides a promoter that encodes driver fusion oncoproteins, which induces cancer by acting on the downstream signaling pathway (7). While ALK rearrangements are associated 

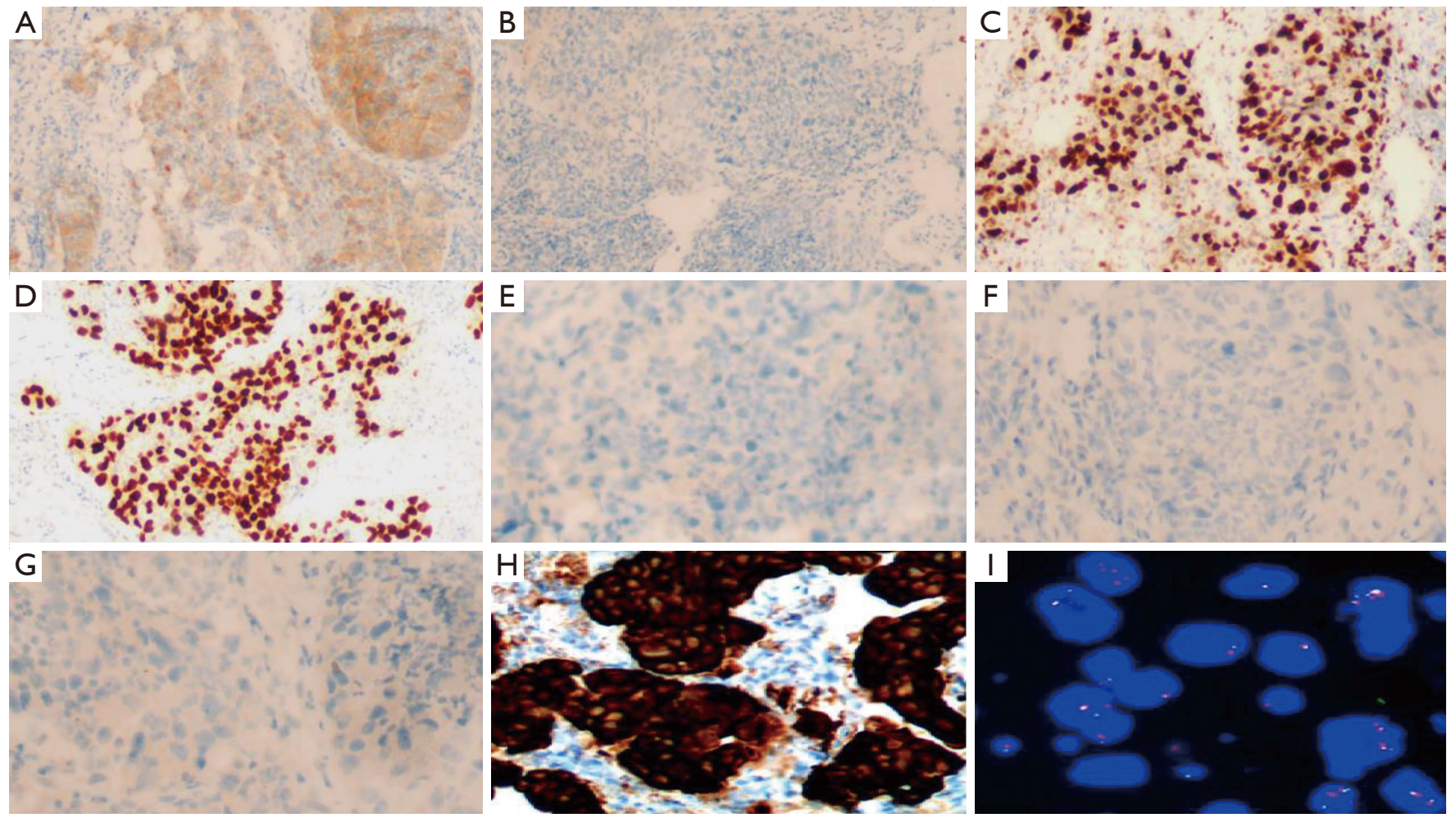

Figure 2 Immunohistochemistry analysis revealed immunoreactivity to CK (A), CK7 (B), Ki-67 (C), TTF-1 (D), CK56 (E), Napsin A (F), and P40 (G). Original magnification of images: $\times 100$ for CK, CK7, Ki-67, TTF-1 and $\times 200$ for CK56, Napsin A, P40 (immunohistochemical staining). (H) Immunohistochemistry staining showed strong ALK receptor tyrosine kinase protein expression (×200). (I) Fluorescent in situ hybridization showed fused, split red-green signals, and single red signals $(\times 100)$. CK, cytokeratin; TTF-1, thyroid transcription factor 1 ; ALK, anaplastic lymphoma kinase.

with unregulated tyrosine kinase activity, TKIs are established effective therapy in the ALK-rearranged subpopulation for prolonged disease control and have been proven superior to chemotherapy in the previous phase III clinical trials $(8,9)$. Given the superior and confirmed efficacy of ALK inhibitors shown in patients with advanced ALK-positive NSCLC, identifying the novel ALK rearrangement is meaningful, both for improving survival outcomes of patients and wiser medical decision-making of oncologists, which underlines the significance of NGS to discover $(10,11)$. Using the NGS technology, which allows sequencing a high number of nucleotides in a brief time frame at an affordable cost, rarer partner genes to ALK have been identified. Also, the new type of variant expanded the spectrum of ALK rearrangement and provided more insight into precise treatment with ALK-TKIs for the maximum benefit to NSCLC patients.

CCNY, a newly identified PFTK1 interacting protein, plays an increasingly important role in the progression of tumor invasion and metastasis and is overexpressed in NSCLC, associated with NSCLC proliferation and tumorigenesis (12). Although there is no direct evidence to support CCNY-ALK as a driver alteration, considering the overexpression of CCNY in samples of NSCLC, there is a possibility that CCNY-ALK rearrangement acts as a driver alteration. 5-aminoimidazole-4-carboxamide ribonucleotide formyltransferase/IMP cyclohydrolase (ATIC) is a common partner. The ribonucleotide comprises approximately $1 \%$ ALK rearrangement in anaplastic large cell lymphomas (ALCL) and inflammatory myofibroblastic tumors (IMT) (13). However, to the best of our knowledge, CCNY-ALK (C1:A20) and ATIC-ALK (A7:A20) had not been reported in NSCLC before. Also, the response of patients with NSCLC harboring the CCNY-ALK and ATIC-ALK to ALK inhibitors is still unknown. However, in our patient, immunohistochemistry reveals strong 


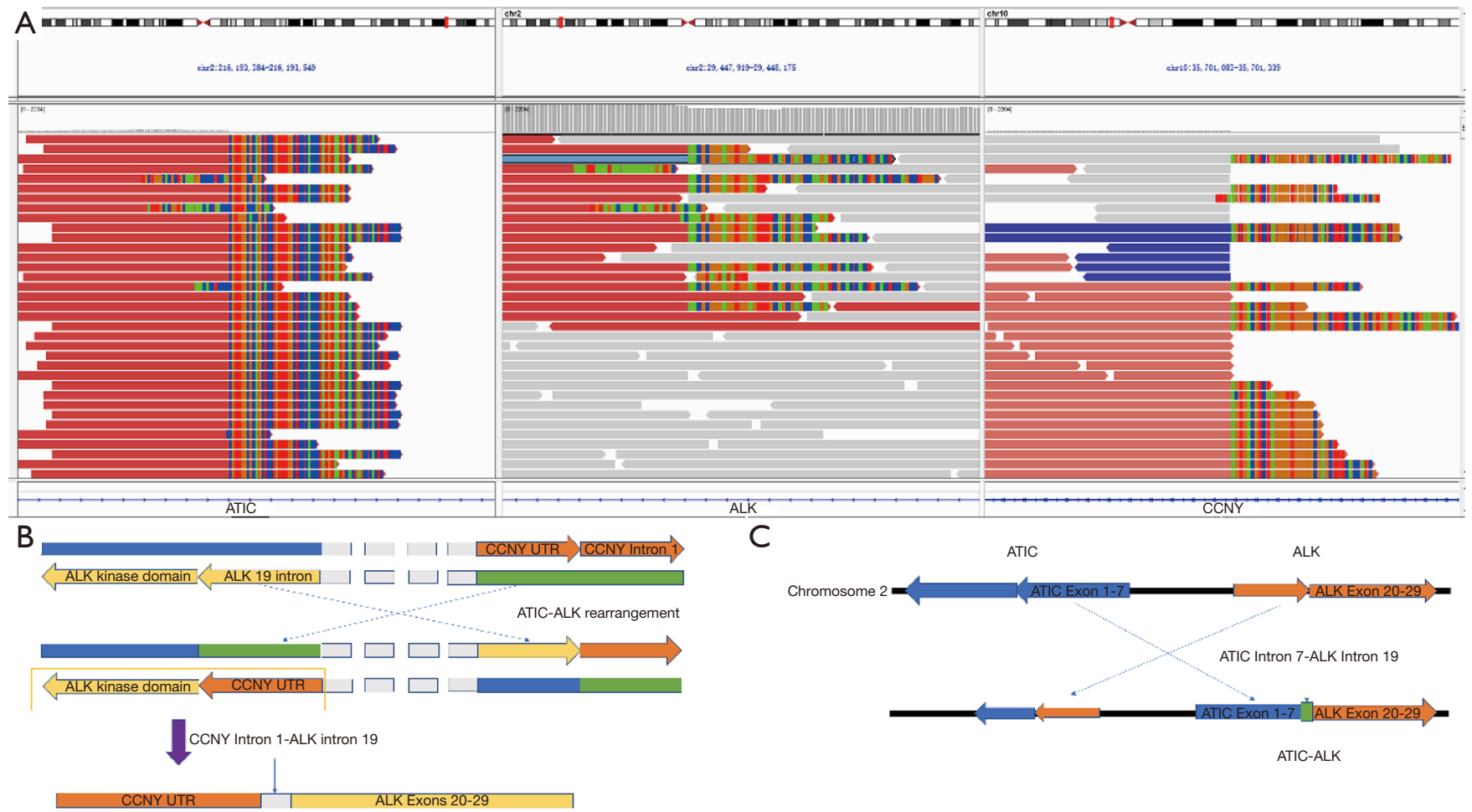

Figure 3 Identification of CCNY-ALK and ATIC-ALK coexists in an NSCLC patient. (A) IGV shows the breakpoints on the ATIC, ALK, and CCNY gene detected by capture-based next-generation sequencing. (B) CCNY-ALK genomic DNA rearrangement and the resulting transcript. (C) ATIC-ALK genomic DNA rearrangement and the resulting transcript. CCNY, cyclin Y; ALK, anaplastic lymphoma kinase; ATIC, 5-aminoimidazole-4-carboxamide ribonucleotide formyltransferase/IMP cyclohydrolase, NSCLC, non-small-cell lung cancer; UTR, untranslated regions; IGV, integrative genomics viewer.

expression of ALK protein in tumor cells. The patient responded well to crizotinib and achieved a partial response for over six months.

\section{Conclusions}

Our study was the first to report two ALK rearrangements, a novel CCNY-ALK and ATIC-ALK, coexisting in one patient with NSCLC responded well to crizotinib. However, whether both CCNY-ALK and ATIC-ALK rearrangement transcripts are sufficient to induce tumor formation is unclear and will be studied in future research.

\section{Acknowledgments}

Funding: The project supported was cosponsored by the Henan Province and Ministry of Health, Medical Science and Technology Program (No. 201601026) and in part by the National Natural Science Foundation of China (No.
81272600), Natural Science Foundation of Henan Province (No. 162300410300), ZHONGYUAN QIANREN JIHUA, and the 51282 projects Leading Talent of Henan Provincial Health Science and Technology Innovation Talents (No. [2016]32). The program also supported it for Science and Technology Innovation Talents in Universities of Henan Province (No. 18HASTIT044). The funders had no role in the study, design, data collection, and analysis, decision to publish, or preparation of the manuscript.

\section{Footnote}

Reporting Checklist: The authors have completed the CARE reporting checklist. Available at http://dx.doi.org/10.21037/ tlcr-20-1049

Conflicts of Interest: All authors have completed the ICMJE uniform disclosure form (available at http://dx.doi. org/10.21037/tlcr-20-1049). The authors have no conflicts 
of interest to declare.

Ethical Statement: The authors are accountable for all aspects of the work in ensuring that questions related to the accuracy or integrity of any part of the work are appropriately investigated and resolved. The Medical Ethics Committee approved this study of Henan Cancer Hospital of Zhengzhou University, and the participating patients gave consent. All procedures performed in studies involving human participants were in accordance with the Helsinki Declaration (as revised in 2013). The patient gave written consent for his personal or clinical details, along with any identifying images to be published in this study. A copy of the written consent is available for review by the Editor of this journal. The case has not been reported before and is not being considered for publication elsewhere.

Open Access Statement: This is an Open Access article distributed in accordance with the Creative Commons Attribution-NonCommercial-NoDerivs 4.0 International License (CC BY-NC-ND 4.0), which permits the noncommercial replication and distribution of the article with the strict proviso that no changes or edits are made and the original work is properly cited (including links to both the formal publication through the relevant DOI and the license). See: https://creativecommons.org/licenses/by-nc-nd/4.0/.

\section{References}

1. Shaw AT, Engelman JA. ALK in lung cancer: past, present, and future. J Clin Oncol 2013;31:1105-11.

2. Delmonte A, Burgio MA, Verlicchi A, et al. New generation anaplastic lymphoma kinase inhibitors. Transl Lung Cancer Res 2019;8:S280-9.

3. Li T, Zhang F, Wu Z, et al. PLEKHM2-ALK: A novel fusion in small-cell lung cancer and durable response to ALK inhibitors. Lung Cancer 2020;139:146-50.

Cite this article as: $\mathrm{Wu} \mathrm{X,} \mathrm{Zhou} \mathrm{H,} \mathrm{He} \mathrm{Z,} \mathrm{Zhang} \mathrm{Z,} \mathrm{Feng} \mathrm{W,}$ Zhao J, Chen H, Wang S, Wang W, Wang Q. Coexistence of a novel CCNY-ALK and ATIC-ALK double-fusion in one patient with ALK-positive NSCLC and response to crizotinib: a case report. Transl Lung Cancer Res 2020;9(6):2494-2499. doi: 10.21037/tlcr-20-1049
4. Ma L, Zhang Q, Dong Y, et al. SPECC1L-ALK: A novel gene fusion response to ALK inhibitors in non-small cell lung cancer. Lung Cancer 2020;143:97-100.

5. He Z, Wu X, Ma S, et al. Next-generation Sequencing Identified a Novel WDPCP-ALK Fusion Sensitive to Crizotinib in Lung Adenocarcinoma. Clin Lung Cancer 2019;20:e548-51.

6. Luo J, Gu D, Lu H, et al. Coexistence of a Novel PRKCB-ALK, EML4-ALK Double-Fusion in a Lung Adenocarcinoma Patient and Response to Crizotinib. J Thorac Oncol 2019;14:e266-8.

7. Katayama R, Lovly CM, Shaw AT. Therapeutic targeting of anaplastic lymphoma kinase in lung cancer: a paradigm for precision cancer medicine. Clin Cancer Res 2015;21:2227-35.

8. Solomon BJ, Mok T, Kim DW, et al. First-line crizotinib versus chemotherapy in ALK-positive lung cancer. N Engl J Med 2014;371:2167-77.

9. Marshall H. Ceritinib versus chemotherapy in ALKpositive lung cancer. Lancet Respir Med 2016;4:952-3.

10. Letovanec I, Finn S, Zygoura P, et al. Evaluation of NGS and RT-PCR Methods for ALK Rearrangement in European NSCLC Patients: Results from the European Thoracic Oncology Platform Lungscape Project. J Thorac Oncol 2018;13:413-25.

11. Shaw AT, Kim DW, Nakagawa K, et al. Crizotinib versus chemotherapy in advanced ALK-positive lung cancer. $\mathrm{N}$ Engl J Med 2013;368:2385-94.

12. Yue $W$, Zhao $X$, Zhang $L$, et al. Cell cycle protein cyclin $\mathrm{Y}$ is associated with human non-small-cell lung cancer proliferation and tumorigenesis. Clin Lung Cancer 2011;12:43-50.

13. Maes B, Vanhentenrijk V, Wlodarska I, et al. The NPMALK and the ATIC-ALK fusion genes can be detected in non-neoplastic cells. Am J Pathol 2001;158:2185-93.

(English Language Editor: J. Chapnick) 